УДК 616.831-005.1/.4-036+615.273.53

DOI: 10.26435/UC.V13I1.264

Ю.И. Коценко, Е.А. Статинова

ГОО ВПО «Донецкий национальный медицинский университет имени М. Горького», Донецк

\title{
СОВРЕМЕННЫЙ ВЗГЛЯД НА СИСТЕМНУЮ ТРОМБОЛИТИЧЕСКУЮ ТЕРАПИЮ В ОСТРЕЙШИЙ ПЕРИОД ИШЕМИЧЕСКОГО МОЗГОВОГО ИНСУЛЬТА
}

Церебральный ишемический инсульт (ЦИИ) является важнейшей медико-социальной проблемой и продолжают оставаться главной причиной госпитализации, инвалидизации и смертности населения [1, 7, 14, 21]. Современные подходы к лечению ЦИИ включают применение высокоэффективных методов церебральной реперфузии в первые часы заболевания, направленных на восстановление кровотока в пораженном сосуде, и предотвращение возможности развития необратимого неврологического дефицита с минимизированием степени его выраженности $[2,14]$.

Согласно рекомендациям Европейской инсультной организации (ESO) (класс доказательности 1, уровень A) и Американской инсультной ассоциации (ASA) (класс доказательности 1, уровень В), системная тромболитическая терапия (ТЛТ) с использованием рекомбинантного тканевого активатора плазминогена (rt-PA) является наиболее эффективным и безопасным методом реперфузионной терапии при ЦИИ в первые 4,5 часа от появления неврологического дефицита [1, 2, 22, 25]. Известно, что в Европе доступными для клинического применения используют устройства для тромбэктомии и сосудистого доступа, которые не одобрены комиссией США по контролю за продовольствием и лекарственными препаратами (FDA) ERIC (MicroVen- tion), EmboTrap I.I. (Neuravi), ClearLumen (Walk Vascular), Lazarus Effect Cover (Medtronic), эффективность которых признана [3-5, 32].

Будущие исследования должны установить новую рекомендацию для обеспечения наилучшей реканализации с меньшим риском не желаемой эмболии. Существует 4 устройства, одобренные FDA для эндоваскулярного лечения острого ишемического инсульта: Merci Retriever (Concentric Medical), Penumbra System (Penumbra), Solitaire F.R. Revascularization Device (Covidien), Trevo (Concentric Medical) [6, 7, 10].

Недостаточная изученность методики оказания помощи пациентам в острейший период ЦИИ, сложность медицинских и диагности- ческих аспектов приводит к необходимости совершенствования терапевтических подходов и определяет актуальность данной проблемы.

\section{ЦЕЛ Ь РАБОТЫ}

Изучить современные данные о механизме, показаниях и противопоказаниях к проведению ТЛТ пациентам в острейшем периоде ЦИИ и оценить его роль в реперфузионной терапии.

Высокий уровень эффективности (класс I, уровень А) и безопасности системного тромболизиса при ЦИИ основан на результатах международных плацебоконтролируемых исследований (NINDS, ECASS I-III, ATLANTIS, PROACT II, MAST I, NEST II, IST-3), нескольких метаанализов и данных международных регистров SITS-MOST и SITS-ISTR [4, 5, 16, 21, 26, 30].

Первое рандомизированное плацебоконтролируемое исследование было выполнено NINDS (National Institute of Neurological Disorders and Stroke), которое доказало безопасность и эффективность системной ТЛТ с использованием rt-РА в первые три часа от момента развития заболевания. В основной группе пациентов, получавших rt-PA, показатель вероятности отсутствия инвалидизации или наличия минимального неврологического дефицита был на 30\% выше, чем в контрольной группе спустя три месяца после ЦИИ. Достоверных различий уровня летальности не выявлено (17\% при использовании rt-РА и $21 \%$ - плацебо, $\mathrm{p}=0,3)$, несмотря на увеличение частоты симптомного внутримозгового кровоизлияния среди обследуемых основной группы в сравнении с контрольной группой $(6,4$ против $0,6 \%, p=0,001)[5,6,9,26]$.

В рандомизированных плацебоконтролируемых исследованиях ECASS (European Cooperative Acute Stroke Study) I и II оценивалась безопасность и эффективность применения rt-PA в разных дозах (ECASS I - 1,1 мг/кг, а ECASS II - 0,9

( Ю.И. Коценко, Е.А. Статинова, 2019

(c) Университетская Клиника, 2019 
мг/кг) в течение шести часов от начала развития ЦИИ. Результаты ECASS I и ECASS II были сопоставимы с результатами NINDS без достоверных различий по эффективности между группами rtРА и плацебо. [5, 6, 26, 30]

В исследованиях ATLANTIS (Alteplase ThromboLysis for Acute Noninterventional Therapy in Ischemic Stroke) А и В оценивалась безопасность и эффективность применения rt-PA в дозе 0,9 мг/кг в течение пяти часов от начала развития ЦИИ. В начале терапии за пятичасовой период не выявлено положительного эффекта от применения rt-РА по сравнению с плацебо. Однако, при получении rt-РА в течение трех часов от начала ЦИИ, увеличивались вероятность благоприятного прогноза спустя 90 дней $(\mathrm{p}=0,01)$, что подтверждает метаанализ исследований NINDS, ECASS I и II. Летальный исход не увеличивался, несмотря на увеличение частоты развития внутричерепных кровоизлияний (по сравнению с плацебо). В ATLANTIS раннее начало ТлТ достоверно приводило к регрессу неврологических нарушений после применения rt-PA до трех часов от начала заболевания и повышался на 50\% по сравнению с плацебо. При этом через 3-4,5 часа от начала заболевания сохранялась вероятность благоприятного исхода (отношение шансов (ОШ) 1,34, 95\% доверительный интервал (ДИ) 1,04-1,72); начиная с 4,5 до шести часов ТЛТ был неэффективен (ОШ 1,04, 95\% ДИ 0,841,29) $[11,12,30]$.

Для оценивания благоприятных функциональных исходов при применении rt-PA в период до 4,5 часов от начала ЦИИ выполняли исследования ECASS III. В результате исследования ECASS III выявлено увеличение случаев регресса неврологического дефицита по сравнению с плацебо (52,4 против 45,2\%, ОШ 1,34, 95\% ДИ 1,0-1,65, p <0,05). При этом внутричерепные кровоизлияния развивались чаще у обследуемых, получавших rt-РА, по сравнению с группой плацебо (27 против 17,6\%, p = 0,001). Достоверных различий количества летальных исходов между группами не выявлено (7,7 против 8,4\%, p $=0,68)$. По результатам ECASS III были пересмотрены Европейские и Американские рекомендации по лечению ЦИИ, в том числе увеличена продолжительность терапевтического окна при проведении системного тромболизиса до 4,5 часов $[5,6,13,31]$.

При многоцентровых исследованиях острого ЦИИ MAST-I, MAST-E (Multicentre Acute Stroke Trial) и ASK (Australian Streptokinase Trial) использовалась стрептокиназа, однако, исследование в группе, получавших стрептокиназу, было досрочно завершено из-за увеличения риска летальности вследствие более высокой частоты ге- моррагической трансформации зоны ишемического инсульта [13, 18, 30].

В исследованиях DEDAS (Dose Escalation of Desmoteplase for Acute Ischemic Stroke) и DIAS (Desmoteplase in Acute Ischemic Stroke) I и II изучался другой фибринолитик (десмотеплаза) более фибринспецифичного и менее нейротоксичного по сравнению с rt-РА. Десмотеплаза вводилась в течение трех - девяти часов от начала ЦИИ при выявлении области перфузионнодиффузионного несоответствия (DWI-PWI mismatch) при магнитно-резонансной томографии (МРТ) головного мозга. В первой части исследования DIAS пациенты получали 25, 37,5 или 50 мг препарата либо плацебо. Исследование было досрочно остановлено из-за высокой частоты симптомных внутричерепных кровоизлияний в группе десмотеплазы (26,7\%). Вторая часть исследования DIAS заключалась в использовании меньших доз препарата (62,5, 90 и 125 мкг/кг), что значительно снизило количество случаев симптомной геморрагической трансформации зоны ишемического инсульта (2,2\%). Реканализация в группе, получавшей десмотеплазу в дозе 125 мкг/кг, наблюдалась в $53,3,4 \%$ случаев, в дозе 90 мкг/кг - у 37,5\% в то время как в группе плацебо -в 18,2\% случаев (р = 0,0012). Благоприятный исход ЦИИ к 90 дню в группе плацебо составлял $22,2 \%$, в группе десмотеплазы в дозе 62,5 мкг/кг -13,3\% (р = 0,757), у 60\% пациентов при введении дозы 125 мкг/кг ( $\mathrm{p}=0,0090)$. B DEDAS показано применение десмотеплазы в интервале трех - девяти часов от начала развития ЦИИ у пациентов с верифицированным перфузионно-диффузионным несоответствием при МРТ ассоциируется с высокой частотой реканализации и хорошими клиническими исходами ЦИИ по сравнению с плацебо $[14,15,19,20,30]$.

При исследовании десмотеплазы в рандомизированном плацебоконтролируемом исследовании DIAS II безопасность и эффективность десмотеплазы не получили подтверждения. В исследовании участвовали 186 пациентов с симптоматикой ЦИИ, где с начала развития неврологического дефицита не прошло девяти часов. Все обследуемые распределены на три группы, где получали десмотеплазу в дозе 95 мкг/ кг $(\mathrm{n}=57)$, в дозе 125 мкг/кг (n=66) или плацебо $(\mathrm{n}=63)$. Благоприятные клинические исходы заболевания к 90 дню в группах пациентов, получивших десмотеплазу в дозах 95 и 125 мкг/ кг, наблюдались в 47\% и 36\% случаев соответственно. В группе плацебо благоприятные клинические исходы к указанному сроку отмечены в 46\% (n=29) случаев. Частота симптомных внутричерепных кровоизлияний при использова- 
нии десмотеплазы в дозах 95 и 125 мкг/кг составила 3,5 и 4,5\% соответственно. В группе плацебо церебральные геморрагические осложнения не отмечалось [16, 17, 29, 30, 31].

В настоящее время проведение ТЛТ является методом дифференцированного лечения ЦИИ в стационаре, имеющем в структуре блок интенсивной терапии и реанимации для лечения больных, при условии обязательного наличия нейровизуализации (компьютерной или высокопольной магнитно-резонансной томографии) и лабораторной диагностики, функционирующих ежедневно круглосуточно, в том числе возможность неотложной консультации врачанейрохирурга [23, 24].

Максимально быстрая транспортировка пациента в стационар, а также сокращение времени обследования для верификации характера инсульта являются залогом дальнейшего успешного лечения, в частности, проведения ТЛТ. Для минимизирования траты времени при поступлении больных с клинической картиной ОНМК необходимы соблюдение отработанного порядка действий персонала, сокращение внутрибольничных перемещений, обязательное следование стандартным диагностическим протоколам, использование разработанных формализованных шкал и алгоритмов (шкала инсультов Национальных институтов здоровья NIHSS, шкала комы Глазго). Данный вариант госпитализации позволяет значительно сократить временной диапазон от поступления до начала ТЛТ, «от двери до иглы» («door-to-needle time») и оптимально должен составлять менее 40 минут или не превышать 60 минут [1, 12, 22, 25, 26].

Перед проведением ТЛТ в течение 20 минут от момента взятия крови, но не позже 40 минут от поступления необходимо выполнить лабораторную диагностику с определением основных показателей гемостаза (тромбоциты, МHO, АЧТВ, ПТВ, ПТИ, ТВ) и с целью исключения сопутствующей тяжелой соматической патологии (метаболические и электролитные нарушения, заболевания крови и внутренних органов) [22, 23, 25].

Для исключения признаков внутричерепного кровоизлияния (субарахноидальное, паренхиматозное и вентрикулярное), а также определения объема и характера ишемических изменений используют нейровизуальзационные методы (КТ и/или МРТ головного мозга). При выявлении признаков ишемического повреждения для оценки объема ишемического очага по результатам КТ в рутинной практике рекомендуется использование шкалы ASPECTS (Alberta Stroke Program Early C.T. Score, 2001) [22, 26, 27].
Протокол ведения ТЛТ предполагает оценку показаний и противопоказаний, соблюдение временного интервала для введения препарата (начало не позднее 4,5 часов после появления первых симптомов), соответствие параметров центральной гемодинамики пациента безопасному уровню (менее 185 и $110 \mathrm{mmHg}$ ) на момент начала терапии. Для обеспечения процедуры системного тромболизиса необходима постановка кубитального периферического венозного катетера [18, 19, 29].

Внутривенное введение rt-РА проводится в условиях отделения (блока) реанимации и интенсивной терапии, где обеспечивается постоянный мониторинг параметров системной гемодинамики, функции дыхания, сатурации кислородом периферической крови, температуры тела, баланса жидкостей [22, 24, 25].

В исследовании NINDS (National Institute of Neurological Disorders and Stroke) дозирование препарата rt-PA (Алтеплаза) проводится индивидуально для каждого пациента с учетом массы тела больного, не превышая максимально допустимой дозы - 90 мг. Первые 10\% дозы rt-PA вводится внутривенно болюсно в течение 1 минуты. Основные 90\% дозы вводится с помощью инфузомата медленно. В период инфузии основной дозы допускается прерывать введение rtРА, однако общая продолжительность введения препарата не более одного часа после введения болюсной части (10\% дозы) в первые 4,5 ч после начала ЦИИ рекомендовано как в европейских (ESO), так и североамериканских (AHA/ASA) руководствах по ведению пациентов с ЦИИ [6, 20, 27, 31].

Исследование NINDS состоит из 2 этапов. На первом этапе первичной конечной точкой был полный регресс неврологической симптоматики (полное «клиническое выздоровление») или уменьшение неврологических нарушений не менее чем на 4 балла по шкале тяжести ЦИИ Национальных институтов здоровья (National Institutes of Health Stroke Scale - NIHSS) (T. Brott et al. 1989, J. Biller et al., 1990) в течение первых 24 часов. На втором этапе первичной финальной точкой являлся полный или выраженный регресс неврологических нарушений спустя 3 месяца после ЦИИ. На первом этапе исследования положительные результаты получены у 31\% пациентов, получавших ТЛТ, и у 20\% больных группы плацебо. На втором этапе исследования положительные результаты были достигнуты у 50\% пациентов, получавших ТЛт, по сравнению с 38\% группы плацебо. Сходные показатели эффективности терапии сохранялись спустя один год после ЦИИ. Несмотря на то, что частота развития симптомных внутримозговых 
кровоизлияний выше у пациентов, получавших лечение рекомбинантным активатором плазминогена rtPA (6,4\% по сравнению с 0,6\% в группе плацебо), такие показатели, как смертность через 3 месяца и через один год после инсульта, были сходными и даже несколько более высокими в группе плацебо (в группе rtPA и плацебо соответственно $17 \%$ и 20\% через 3 месяца; $24 \%$ и 28\% через 1 год). В ходе исследования отмечено достоверное снижение суммарного уровня смертности и инвалидности и недостоверное смертности за весь период наблюдения у пациентов, получавших ТЛТ $[1,5,6,13,20]$.

На протяжении всего периода введения препарата и в течение ближайших 24 часов осуществляется динамическое наблюдение за состоянием пациента, возможными побочными явлениями и осложнениями. По NIHSS необходимо оценивать неврологический статус и витальные функции (частоту пульса и дыхания, сатурацию крови кислородом, температуру тела, артериальное давление - АД) каждые 15 минут в процессе введения rt-PA, каждые 30 минут в последующие 6 часов и каждый час до истечения 24 часов после введения препарата. Контролировать АД каждые 15 минут в первые 2 часа, каждые 30 минут последующие 6 часов и каждый час до истечения 24 часов после введения препарата, где целевые показатели АД не выше 180/105 mmHg [6, 14, 30].

В первые сутки после ТЛТ необходимо воздержаться от использования назогастральных зондов, мочевых, внутрисосудистых катетеров и не допускать применения антиагрегантных и антикоагулянтных средств в ближайшие 24 часа после проведения системного тромболизиса. С целью снижения риска возникновения травм и кровоизлияний, в течение 24 часов после введения препарата больной не должен подвергаться перекладыванию и дополнительным перемещениям из отделения реанимации и интенсивной терапии, за исключением ситуаций экстренной необходимости [2, 3, 7, 32].

К противопоказаниям ТЛТ относят неизвестное время появления первых симптомов мозговой катастрофы или «терапевтическое окно» больше 4,5 часов, легкий (NIHSS $\leqslant 4$ баллов) или тяжелый (NIHSS > 25) неврологический дефицит, наличие менингеальных знаков, как подозрение на субрахноидальное кровоизлияние повышение АД свыше 185/110 mmHg., в анамнезе перенесенный геморрагический инсульт, черепномозговая травма, хирургические вмешательства за последние 2 недели, судорожный синдром, при нейровизуализации признаки внутричерепного кровоизлияния, опухоли мозга, абсцесса мозга, артериовенозной мальформации, аневризмы церебральных сосудов, наличия очага ишемии более $1 / 3$ бассейна СMA, тяжелая соматическая патология, желудочно-кишечные и мочеполовые кровотечения за последние 3 недели, беременность, гипогликемия (менее 2,7 ммоль/л), гипергликемия (более 22 ммоль/л), тромбоцитопения (менее 100 Г/л), применение прямых антикоагулянтов [15, 18, 19].

При проведении ТЛТ среди противопоказаний определяются особые ситуации (off-label), применяемые в специализированных центрах с достаточным опытом выполнения ТЛТ и наличием возможности использования эндоваскулярных вмешательств. Применение внутривенной ТЛТ в каждом конкретном случае должно быть основано на оценке соотношения риска процедуры и потенциальной пользы для пациента. К основным показаниям off-label относят возраст старше 80 лет, лечение вне «терапевтического окна», минимальный неврологический дефицит менее 4 баллов по NIHSS, судороги в дебюте болезни, сахарный диабет и ЦИИ в анамнезе [2, 17, 22, 25, 28].

Критериями эффективности ТЛТ определяется успешная реканализация и реперфузия в пораженном сосудистом бассейне, клинической динамикой и развитием осложнений, в частности геморрагической трансформации очага поражения головного мозга. Положительная динамика в течение первых 24 часов после проведения ТЛТ, как правило, позволяет прогнозировать последующее хорошее восстановление [19, 21].

Случаи клинического улучшения с уменьшением балла по шкале инсульта NIHSS на 4 и более через 24 часа после начала ТЛТ могут расцениваться как значительное, или «драматическое», улучшение. Конечные исходы оцениваются по модифицированной шкале Рэнкина, согласно которой 0-1 балл соответствует благоприятному исходу, а 0-2 балла - выходу на независимость в повседневной жизни. Реканализация инфарктзависимой артерии после проведения ТЛТ верифицируется на основании данных ультразвуковых методов исследования, а также данных МРангиографии или КТ-ангиографии [7, 20, 22].

\section{З АКЛЮЧЕНИЕ}

При правильной интерпретации неврологического дефицита, оценке показаний и противопоказаний к проведению ТЛТ, а также скорости выполнения необходимого объема подготовительных мероприятий, максимально минимизирует риск развития осложнений. Самой частой неблагоприятной реакцией, связанной с введением фибринолитиков, являются кровоизлияния, приводящие к снижению гематокрита и (или) гемоглобина. 
Клинические факторы, коррелирующие с риском осложнений ТЛТ у больных с ЦИИ, являются пожилой возраст, тяжёлый инсульт (более 20 баллов по шкале NIHSS), симптом гиперденсивной СМА по данным КТ-исследования головного мозга, гипергликемия. Однако, ни один из перечисленных факторов не уменьшает вероятную пользу от проведения ТЛТ и не является абсолютным противопоказанием для отказа от проведения реперфузионной терапии.

\section{Ю.И. Коценко, Е.А. Статинова}

ГОО ВПО «Донецкий национальный медицинский университет им. М. Горького», Донецик

\section{СОВРЕМЕННЫЙ ВЗГЛЯД НА СИСТЕМНУЮ ТРОМБОЛИТИЧЕСКУЮ ТЕРА- ПИЮ В ОСТРЕЙШИЙ ПЕРИОД ИШЕМИЧЕСКОГО МОЗГОВОГО ИНСУЛЬТА}

Реперфузионная терапия при церебральном ишемическом инсульте увеличивает вероятность благоприятного прогноза в виде снижения летальности и улучшения качества жизни, профилактики повторной мозговой катастрофы и венозных тромбозов. Цель работы в статье изучить современные данные о меха- низме, показаниях и противопоказаниях к проведению тромболитической терапии пациентам в острейшем периоде церебрального ишемического инсульта и оценить его роль в реперфузионной терапии.

Ключевые слова: ишемический инсульт, тромболизис, лечение.

\section{Yu.I. Kotsenko, E.A. Statinova}

SEI HPE «M. Gorky Donetsk National Medical University», Donetsk

\section{MODERN VIEW ON SYSTEMIC THROMBOLYTIC THERAPY OF ACUTE CEREBRAL ISCHEMIC STROKE}

Reperfusion therapy for cerebral ischemic stroke increases the chance of a favorable prognosis in the form of mortality reduction and improved quality of life, prevention of recurrent cerebral disaster and venous thrombosis. The purpose of the work in the article is to study the current data on the mechanism, indications and contra indications for carrying out thrombolytic therapy in patients with acute cerebral ischemic stroke and assess its role in reperfusion therapy.

Key words: ischemic stroke, thrombolysis, treatment.

\section{ЛИТЕРАТУРА}

1. Lansberg M.G., O’Donnell M.J., Khatri P. [et al.] Antithrombotic and thrombolytic therapy for ischemic stroke: Antithrombotic Therapy and Prevention of Thrombosis, 9th ed: American College of Chest Physicians Evidence-Based Clinical Practice Guidelines. Chest, 2012; 141: 601-636.

2. Клинические рекомендации по проведению тромболитической терапии у пациентов с ишемическим инсультом. Москва; 2015. 34. URL: https://mosgorzdrav.ru/ ru-RU/science/default/download/86.html (дата обращения: 17.11.2018)

3. Скворцова В.И., Волынский Ю.Д., Шамалов Н.А. [и др.] Организационные аспекты проведения внутриартериального тромболизиса при ишемическом инсульте. Новости медицины и фармации. Неврология. 2010; 328: $15-18$.

4. Del Zoppo G.J., Saver J.L., Jauch E.C., Adams H.P. Expansion of the time window for treatment of acute ischemic stroke with intravenous tissue plasminogen activator: a science advisory from the American Heart Association/ American Stroke Association. Stroke. 2009; 40 (8): 29452948.

5. Powers W.J., Derdeyn C.P., Biller J. AHA/ASA Guideline Focused Update of the 2013 Guidelines for the Early Management of Patients With Acute Ischemic Stroke Regarding Endovascular Treatment: A Guideline for Healthcare Professionals From the American Heart Association/American Stroke Association. Stroke. 2015; Vol. 46: 3020-3035.

6. Powers W.J., Rabinstein A.A., Ackerson T. Guidelines for the Early Management of Patients With Acute Ischemic

\section{REFERENCES}

1. Lansberg M.G., O’Donnell M.J., Khatri P. [et al.] Antithrombotic and thrombolytic therapy for ischemic stroke: Antithrombotic Therapy and Prevention of Thrombosis, 9th ed: American College of Chest Physicians Evidence-Based Clinical Practice Guidelines. Chest, 2012; 141: 601-636.

2. Klinicheskie rekomendatsii po provedeniyu tromboliticheskoi terapii u patsientov s ishemicheskim insul'tom. Moskva; 2015. 34. URL: https://mosgorzdrav.ru/ru-RU/ science/default/download/86.html (data obrashcheniya: 17.11.2018) (in Russian).

3. Skvortsova V.I., Volynskii Yu.D., Shamalov N.A. [i dr.] Organizatsionnye aspekty provedeniya vnutriarterial'nogo trombolizisa pri ishemicheskom insul'te. Novosti meditsiny i farmatsii. Nevrologiya. 2010; 328: 15-18 (in Russian).

4. Del Zoppo G.J., Saver J.L., Jauch E.C., Adams H.P. Expansion of the time window for treatment of acute ischemic stroke with intravenous tissue plasminogen activator: a science advisory from the American Heart Association/ American Stroke Association. Stroke. 2009; 40 (8): 29452948.

5. Powers W.J., Derdeyn C.P., Biller J. AHA/ASA Guideline Focused Update of the 2013 Guidelines for the Early Management of Patients With Acute Ischemic Stroke Regarding Endovascular Treatment: A Guideline for Healthcare Professionals From the American Heart Association/American Stroke Association. Stroke. 2015; Vol. 46: 3020-3035.

6. Powers W.J., Rabinstein A.A., Ackerson T. Guidelines for the Early Management of Patients With Acute Ischemic 
Stroke: A Guideline for Healthcare Professionals From the American Heart Association/American Stroke Association Stroke. 2018; Vol. 49, 3: 46-110.

7. Инсульт: Руководство для врачей. Под редакцией Стаховской Л.В., Котова С.В. Издательство МИА; 2018. 488.

8. Rivkin M.J., deVeber G., Ichord R.N. [et al.] Thrombolysis in pediatric stroke study. Stroke. $2015 ; 46$ (3): 880-885. doi:10.1161/STROKEAHA.114.008210

9. Кадомцев Д.В., Пасечникова Е.А., Занин С.А., Кочарян В.Э., Плотникова В.В., Виноградов И.О. Тромболитическая терапия при ишемическом инсульте. Современное состояние проблемы. Современные проблемы науки и образования. 2016; 4. URL: http://www.scienceeducation.ru/ru/article/view?id=24926 (дата обращения: 17.11.2018).

10. Риччи С., Диния Л., Дел Сетте М. и др. Тромболизис с поддержкой ультразвуком при остром ишемическом инсульте. Журнал национальной ассоциации по борьбе с инсультом /Stroke/ Российское издание. 2013; 1 (29): 72-73.

11. Порембская О.Я., Хмельникер С.М., Шайдаков Е.В. Эндогенный венозный тромболизис. Ангиология и сосудистая хирургия. 2015; Т. 21, 3: 83-88.

12. Manawadu D., Bodla S., Keep J., Jarosz J., Kalra L. An observational study of thrombolysis outcomes in wake-up ischemic stroke patients. Stroke. 2013; 44: 427-431.

13. Нетлюх А.М., Шевага В.М., Паєнок А.В., Сало В.М., Кобилецький О.Я. Сучасні клінічні рекомендації щодо ендоваскулярного лікування пацієнтів 3 гострим ішемічним інсультом та практичні аспекти їх застосування. Ендоваскулярна нейрорентгенохірургія. 2018; 2 (24): 20-29.

14. Скворцова В.И., Шетова И.М., Какорина Е.П. и др. Снижение смертности от острых нарушений мозгового кровообращения в результате реализации комплекса мероприятий по совершенствованию медицинской помощи пациентам с сосудистыми заболеваниями в Российской Федерации. Профилактическая медицина. 2018; T. 21, 1: 4-10.

15. Максимова М.Ю., Фонякин А.В., Гераскина Л.А. Стратегии антитромботической терапии при ишемическом инсульте. Неврология, нейропсихиатрия, психосоматика. 2017; 9 (2): 58-64. doi: 10.14412/2074-27112017-2-58-64

16. Шамалов Н. Реперфузионная терапия при ишемическом инсульте в Российской Федерации: проблемы и перспективы. Неврология, нейропсихиатрия, психосоматика. 2014; 6 (2S):15-22. doi:10.14412/2074-27112014-2S-15-22

17. Guillan M., Alonso-Canovas A., Garcia-Caldentey J. [et al.] Off-label intravenous thrombolysis in acute stroke. Eur I Neurol. 2012; 19 (3): 390-394. doi:10.1111/j.1468-1331.

18. Broderick J.P., Palesch Y.Y., Demchuk A.M. [et al.] Endovascular therapy after intravenous t-PA versus t-PA alone for stroke. N Engl J Med. 2013; 368 (10): 893-903. doi: 10.1056/NEJMoa1214300

19. Тсивгоулис Г., Сафоурис А. Внутривенный тромболизис у пациентов с острым ишемическим инсультом, получавших пероральные антикоагулянты не антагонисты витамина К. Журнал Национальной ассоциации по борьбе с инсультом /Stroke/ Российское издание. 2017; 3 (45): 48-51.

20. Логвиненко Р.Л., Домашенко М.А., Францевич А.М. и др. О выборе реперфузионной стратегии в острейшем периоде ишемического инсульта. Диагностическая и интервенционная радиология. 2018; Т. 12, 2: 77-84.

21. Шамалов Н.А., Гордеев М.Н., Анисимова А.В. и др. Не упустить драгоценное время. Как работает система оказания помощи больным с острыми нарушениями мозгового кровообращения в Москве. Московская медицина. 2018; 1 (22): 74-79.

22. Коломенцев С.В., Одинак М.М., Вознюк И.А. и др. Ишемический инсульт у стационарного пациента. Современный взгляд на состояние проблемы. Вестник Российской военно-медицинской академии. 2017; 2 (58): 206-212.
Stroke: A Guideline for Healthcare Professionals From the American Heart Association/American Stroke Association Stroke. 2018; Vol. 49, 3: 46-110.

7. Insul't: Rukovodstvo dlya vrachei. Pod redaktsiei Stakhovskoi L.V., Kotova S.V. Izdatel'stvo MIA; 2018. 488 (in Russian).

8. Rivkin M.J., deVeber G., Ichord R.N. [et al.] Thrombolysis in pediatric stroke study. Stroke. 2015; 46 (3): 880-885 doi:10.1161/STROKEAHA.114.008210

9. Kadomtsev D.V., Pasechnikova E.A., Zanin S.A., Kocharyan V.E., Plotnikova V.V., Vinogradov I.O. Tromboliticheskaya terapiya pri ishemicheskom insul'te. Sovremennoe sostoyanie problemy. Sovremennye problemy nauki i obrazovaniya. 2016; 4. URL: http://www.science-education.ru/ $\mathrm{ru} /$ article/view?id=24926 (data obrashcheniya: 17.11 .2018 ) (in Russian).

10. Richchi S., Diniya L., Del Sette M. i dr. Trombolizis s podderzhkoi ul'trazvukom pri ostrom ishemicheskom insul'te. Zhurnal natsional'noi assotsiatsii po bor'be $s$ insul'tom / Stroke/ Rossiiskoe izdanie. 2013; 1 (29): 72-73.

11. Porembskaya O.Ya., Khmel'niker S.M., Shaidakov E.V. Endogennyi venoznyi trombolizis. Angiologiya i sosudistaya khirurgiya. 2015; T. 21, 3: 83-88 (in Russian).

12. Manawadu D., Bodla S., Keep J., Jarosz J., Kalra L. An observational study of thrombolysis outcomes in wake-up ischemic stroke patients. Stroke. 2013; 44: 427-431.

13. Netlyukh A.M., Shevaga V.M., Paenok A.V., Salo V.M., Kobilets'kii O.Ya. Suchasni klinichni rekomendatsiï shchodo endovaskulyarnogo likuvannya patsientiv z gostrim ishemichnim insul'tom ta praktichni aspekti ïkh zastosuvannya. Endovaskulyarna neirorentgenokhirurgiya. 2018; 2 (24): 20-29 (in Ukrainian).

14. Skvortsova V.I., Shetova I.M., Kakorina E.P. i dr. Snizhenie smertnosti ot ostrykh narushenii mozgovogo krovoobrashcheniya $\mathrm{v}$ rezul'tate realizatsii kompleksa meropriyatii po sovershenstvovaniyu meditsinskoi pomoshchi patsientam s sosudistymi zabolevaniyami v Rossiiskoi Federatsii. Profilakticheskaya meditsina. 2018; T. 21, 1: 4-10 (in Russian).

15. Maksimova M.Yu., Fonyakin A.V., Geraskina L.A. Strategii antitromboticheskoi terapii pri ishemicheskom insul'te. Nevrologiya, neiropsikhiatriya, psikhosomatika. 2017; 9 (2): 58-64. doi: $10.14412 / 2074-2711-2017-2-58-64$ (in Russian).

16. Shamalov N. Reperfuzionnaya terapiya pri ishemicheskom insul'te v Rossiiskoi Federatsii: problemy i perspektivy. Nevrologiya, neiropsikhiatriya, psikhosomatika. 2014; 6 (2S):15-22. doi:10.14412/2074-2711-2014-2S-1522 (in Russian).

17. Guillan M., Alonso-Canovas A., Garcia-Caldentey J. [et al.] Off-label intravenous thrombolysis in acute stroke. Eur I Neurol. 2012; 19 (3): 390-394 doi:10.1111/i.1468-1331.

18. Broderick J.P., Palesch Y.Y., Demchuk A.M. [et al.] Endovascular therapy after intravenous t-PA versus t-PA alone for stroke. N Engl J Med. 2013; 368 (10): 893-903. doi: 10.1056/NEJMoa1214300

19. Tsivgoulis G., Safouris A. Vnutrivennyi trombolizis $u$ patsientov s ostrym ishemicheskim insul'tom, poluchavshikh peroral'nye antikoagulyanty ne antagonisty vitamina K. Zhurnal Natsional'noi assotsiatsii po bor'be $\mathrm{s}$ insul'tom /Stroke/ Rossiiskoe izdanie. 2017; 3 (45): 48-51 (in Russian).

20. Logvinenko R.L., Domashenko M.A., Frantsevich A.M. i dr. O vybore reperfuzionnoi strategii v ostreishem periode ishemicheskogo insul'ta. Diagnosticheskaya i interventsionnaya radiologiya. 2018; T. 12, 2: 77-84 (in Russian).

21. Shamalov N.A., Gordeev M.N., Anisimova A.V. i dr. Ne upustit' dragotsennoe vremya. Kak rabotaet sistema okazaniya pomoshchi bol'nym s ostrymi narusheniyami mozgovogo krovoobrashcheniya v Moskve. Moskovskaya meditsina. 2018; 1 (22): 74-79 (in Russian).

22. Kolomentsev S.V., Odinak M.M., Voznyuk I.A. i dr. Ishemicheskii insul't u statsionarnogo patsienta. Sovremennyi vzglyad na sostoyanie problemy. Vestnik Rossiiskoi voenno-meditsinskoi akademii. 2017; 2 (58): 206-212 (in Russian). 
23. Максимов Р.С., Деомидов Е.С., Нестерин К.В. Тромболитическая терапия при ишемическом инсульте. Academy. 2017; T. 1, 6 (21): 84-88.

24. Мартинчук Ю.М. Особливості лікування атеротромботичного i кардіоемболічного підтипів ішемічного інсульту із застосуванням тромболітичної терапії: дис. ...канд. мед. наук: 14.01.15. Київ; 2016. 196.

25. Уніфікований клінічний протокол медичної допомоги: Системний тромболізис при ішемічному інсульті (екстрена, вторинна (спеціалізована) медична допомога). 2012. 46. URL:http://mtd.dec.gov.ua/images/dodat ki/2012_602/2012_602dod3ykpmd.pdf (дата обращения: 17.11.2018).

26. Chartrain A.G. Novel and emerging technologies for endovascular thrombectomy. Neurosurg. Focus. 2017; 42 (4): 12.

27. Максимов Р.С., Дмитриева Р.В., Рыбкина О.А., Васильева А.Ю., Николаева О.В. Системный тромболизис при инфаркте мозга. В сборнике: Актуальные проблемы современной медицины Материалы Республиканской научно-практической конференции. Под редакцией Карзаковой Л.М. 2017: 92-98.

28. Breuer L., Blinzler C., Huttner H.B. [et al.] Off-label thrombolysis for acute ischemic stroke: rate, clinical outcome and safety are influenced by the definition of 'minor stroke'. Cerebrovasc Dis. 2011; 32 (2): 177-185. doi:10.1159/000328811

29. Diener H.C., Bernstein R., Butcher K., Campbell B., Cloud G., Davalos A. [et al.] Thrombolysis and thrombectomy in patients treated with dabigatran with acute ischemic stroke: Expert opinion. Int J Stroke. 2017; 12: 9-12. doi:10.1177/1747493016669849

30. Домашенко М.А., Панова К.В., Муртазалиева Д.М. и др. Персонификация тромболитической терапии пациентов с ишемическим инсультом. Medica mente/Лечим с умом. 2017; 3 (1): 45-48.

31. Yaghi S., Boehme A.K., Dibu J. [et al.] Treatment and Outcome of Thrombolysis-Related Hemorrhage: A Multicenter Retrospective Study. JAMA Neurol. 2015; 72 (12): 1451-1457.

32. Bracard S., Ducrocq X., Mas J.L., [et al.] Mechanical thrombectomy after intravenous alteplase versus alteplase alone after stroke (THRACE): a randomised controlled trial. Lancet Neurol. 2016; 15 (11): 1138-1147.
23. Maksimov R.S., Deomidov E.S., Nesterin K.V. Tromboliticheskaya terapiya pri ishemicheskom insul'te. Academy. 2017; T. 1, 6 (21): 84-88 (in Russian).

24. Martinchuk Yu.M. Osoblivosti likuvannya aterotrombotichnogo i kardioembolichnogo pidtipiv ishemichnogo insul'tu iz zastosuvannyam trombolitichnoï terapiï: dis.... kand. med. nauk: 14.01.15. Kiïv; 2016. 196 (in Ukrainian).

25. Unifikovanii klinichnii protokol medichnoï dopomogi: Sistemnii trombolizis pri ishemichnomu insul'ti (ekstrena, vtorinna (spetsializovana) medichna dopomoga). 2012. 46. URL:http://mtd.dec.gov.ua/images/dodatki/2012 602/20 12_602dod3ykpmd.pdf (data obrashcheniya: 17.11.2018) (in Ukrainian).

26. Chartrain A.G. Novel and emerging technologies for endovascular thrombectomy. Neurosurg. Focus. 2017; 42 (4): 12.

27. Maksimov R.S., Dmitrieva R.V., Rybkina O.A., Vasil'eva A.Yu., Nikolaeva O.V. Sistemnyi trombolizis pri infarkte mozga. V sbornike: Aktual'nye problemy sovremennoi meditsiny Materialy Respublikanskoi nauchno-prakticheskoi konferentsii. Pod redaktsiei L.M. Karzakovoi. 2017: 92-98 (in Russian).

28. Breuer L., Blinzler C., Huttner H.B. [et al.] Off-label thrombolysis for acute ischemic stroke: rate, clinical outcome and safety are influenced by the definition of 'minor stroke'. Cerebrovasc Dis. 2011; 32 (2): 177-185. doi:10.1159/000328811

29. Diener H.C., Bernstein R., Butcher K., Campbell B., Cloud G., Davalos A. [et al.] Thrombolysis and thrombectomy in patients treated with dabigatran with acute ischemic stroke: Expert opinion. Int J Stroke. 2017; 12: 9-12. doi:10.1177/1747493016669849

30. Domashenko M.A., Panova K.V., Murtazalieva D.M. i dr. Personifikatsiya tromboliticheskoi terapii patsientov $\mathrm{s}$ ishemicheskim insul'tom. Medica mente/Lechim s umom. 2017; 3 (1): 45-48 (in Russian).

31. Yaghi S., Boehme A.K., Dibu J. [et al.] Treatment and Outcome of Thrombolysis-Related Hemorrhage: A Multicenter Retrospective Study. JAMA Neurol. 2015; 72 (12): 1451-1457.

32. Bracard S., Ducroca X., Mas J.L., [et al.] Mechanical thrombectomy after intravenous alteplase versus alteplase alone after stroke (THRACE): a randomised controlled trial. Lancet Neurol. 2016; 15 (11): 1138-1147. 